\title{
Approach to prevention of non-alcoholic fatty liver disease after liver transplantation
}

\author{
Praveen Sharma, Anil Arora \\ Department of Gastroenterology \& Hepatology, Sir Ganga Ram Hospital, New Delhi, India \\ Contributions: (I) Conception and design: All authors; (II) Administrative support: None; (III) Provision of study material or patients: None; (IV) \\ Collection and assembly of data: None; (V) Data analysis and interpretation: None; (VI) Manuscript writing: All authors; (VII) Final approval of \\ manuscript: All authors. \\ Correspondence to: Dr. Anil Arora. Professor, Department of Gastroenterology, Sir Ganga Ram Hospital, New Delhi, India. \\ Email: dranilarora50@gmail.com.
}

\begin{abstract}
Non-alcoholic fatty liver disease (NAFLD) is one of the most common causes of liver disease and non-alcoholic steatohepatitis (NASH) related cirrhosis is third common indication for liver transplantation (LT). Patients who have NASH related cirrhosis and are candidates for LT often have multiple comorbidities. These comorbidities need to be addressed before and after transplantation as it affects overall survival. Like hepatitis B, hepatitis C, primary biliary cirrhosis, autoimmune hepatitis which recurs after transplantation, NASH also recurs after transplant however the impact of the recurrence on allograft and patient outcomes is unclear. Limited data suggests that it does not affect graft and patient survival. De novo NAFLD which is defined as occurrence of fatty liver in a patient who did not have fatty liver prior to LT can also occur in the allograft of patients transplanted for non-NAFLD liver disease. Obesity, hyperlipidemia, diabetes as well as steroid dose and duration after LT are common predictors of recurrence of NAFLD after transplantation. Studies on prevention and treatment of NASH in post-transplant patients are lacking. Prevention of weight gain, regular exercises, weight reducing surgery, limited steroid use or steroid free regimen have been tried with varying success. Future studies for the prevention of NAFLD/NASH are required especially in post liver transplant patient.
\end{abstract}

Keywords: Non-alcoholic fatty liver; post liver transplant; prevention; treatment

Received: 14 July 2019; Accepted: 15 October 2019; Published: 05 October 2020.

doi: $10.21037 / \operatorname{tgh} .2020 .03 .02$

View this article at: http://dx.doi.org/10.21037/tgh.2020.03.02

Non-alcoholic fatty liver disease (NAFLD) represents a spectrum of disease which is histologically defined as steatotic liver disease that occurs in the absence of significant alcohol consumption. Significant alcohol consumption is defined as $>21$ standard drinks per week in men and $>14$ standard drinks per week in women. This definition is universally accepted for taking NASH patients in trial. Mild disease (non-alcoholic fatty liver) involves mild macrovesicular steatosis without inflammation, whereas severe disease is characterised histologically by hepatocyte ballooning and lobular inflammation (NASH). Severe disease with fibrosis lead to end-stage liver fibrosis or cirrhosis (1). Prevalence of NAFLD varies worldwide and ranges from $20-40 \%$ depending upon the criteria used for diagnosis like ultrasound/impaired liver functions and or biopsy. Its prevalence increases to $60-70 \%$ among the obese population in whom the mortality is also high (2-4). For the diagnosis of NAFLD histological evaluation remains the gold standard both in pretransplant and post-transplant patients. However, biopsy is seldom done in majority of centres due to poor patient compliance, limited treatment options, procedural risks, and cost.

NASH related cirrhosis is now one of the commonest causes of liver disease all over the world and the second commonest cause of LT in developed countries (5). Patients transplanted for NASH related cirrhosis have higher risk 
of recurrence of NASH related liver disease compared to patients with other etiology due to persistence of their previous metabolic risk factors which are often exacerbated by immunosuppressive treatments used after liver transplantation (LT) (5-7).

\section{Post liver transplant recurrence or de novo NAFLD}

The development of fatty liver (steatosis) in post-LT in patients is common (8-10). LT resolves the complications of NASH-related cirrhosis however the metabolic risk factors for NASH do persist and even tend to increase after LT due to immunosuppression with steroids and calcineurin inhibitors (CNI). The definition of recurrence versus de novo disease requires identification of preexisting NAFLD/NASH, in patients undergoing LT. If NASH was diagnosed before the LT and liver shows steatosis after LT it is considered as recurrence and if there was no steatosis prior to LT and it appears for the first time after LT it is considered as de novo NAFLD. It is often under recognized or under reported due to lack of histology prior to LT. There are no histologic features that can differentiate recurrent from de novo NAFLD/NASH after LT. However, these two forms of liver steatosis carry different prognosis and hence should be categorised separately (11).

The development of steatosis posttransplant is a risk factor for NASH and hepatic fibrosis. In a study by Malik et al. (12) 98 patients with NASH related cirrhosis underwent LT. Recurrence of steatosis was seen in $70 \%$ of the recipient, of this one fourth (25\%) had recurrent NASH and $18 \%$ had stage II/IV or greater fibrosis at a mean of 18 months follow-up. Patients with recurrent NASH had a higher incidence of diabetes, weight gain, and dyslipidemia at the time of diagnosis of recurrence and no other specific risk factor were identified related to donor or recipient. Similar were the results by Dureja et al. (8) who analyzed posttransplant data in patients $(\mathrm{n}=88)$ who underwent transplant for NAFLD. This study reported prevalence of recurrent NAFLD to be $39 \%$, recurrent NASH to be $28.4 \%$ and fibrosis (stage 3 and 4 ) to be $3.4 \%$ respectively.

There is paucity of data on the prevalence of de novo NAFLD/ NASH in patients who undergo LT for reason other than NASH related cirrhosis. In a retrospective study by Galvin et al. (13), 430 post-transplant patients were evaluated for de novo steatosis. They excluded patients with NASH related cirrhosis. They found over one third (33\%, $\mathrm{n}=143$ ) had evidence of de novo steatosis and/or NASH at a median of 3 years after liver transplant. Modifiable risk factors associated with development of de novo NAFLD were diabetes, BMI, weight gain after LT, and sirolimus-based immunosuppression. In another study Vallin et al. (11) compared recurrent NAFLD and patients with de novo NAFLD. During follow-up of 5 years posttransplant, severe fibrosis (stage 3 or 4) and steatohepatitis were significantly more in patients with recurrent NAFLD compared to patients with de novo NAFLD. This study suggests that recurrent NAFLD and de novo NAFLD after LT are two different entities and recurrent NAFLD appears to be a more severe and irreversible disease than de novo NAFLD. Effect of recurrent NAFLD on graft survival was analysed by Yalamanchili et al. (14) and it was concluded recurrence of NAFLD after transplant does not have much impact on graft survival compared to LT for other aetiologies.

\section{Risk factors for the development of NAFLD post liver transplant}

Hepatic steatosis is considered as the hepatic manifestation of the metabolic syndrome (MS). Majority of patients (80-90\%) with NAFLD without LT and $50-60 \%$ of posttransplant patients with hepatic steatosis meet one of the criteria for MS $(6,15)$.

Factors related to recurrence of NAFLD posttransplant can be related to recipient factor, environment factor, genetic factors and immunosuppressive factors (Table 1). The main risk for the recurrence of hepatic steatosis appears to be the ongoing metabolic derangements which include insulin resistance or diabetes, hyperlipidemia, and obesity. These risk factors often get exacerbated by immunosuppressive agents like steroids and CNI post LT. Finally, in few studies steatosis in donor liver has been shown to influence both the occurrence and progression of NAFLD in LT patients in few studies (6,16-20).

Immunosuppressive agents used post LT plays a significant role in MS in patients with NASH related LT. Insulin resistance which is a key component in MS is promoted by corticosteroids as it decreases peripheral glucose absorption, increase hepatic glucose production thereby increases risk of post-transplant diabetes. CNI, including cyclosporine and tacrolimus, are also diabetogenic in nature (20). Both steroid and CNI enhance the incidence of hypertension and dyslipidemia, promoting fatty liver in the graft liver. Sirolimus increases triglyceride production and causes insulin resistance and thereby increased the risk 
Table 1 Risk factors for post liver transplant NAFLD

\begin{tabular}{llll}
\hline Host factors & Environment factors & Immunosuppressive factors & Genetic factors \\
\hline Pre-transplant obesity & Lack of exercises/sedentary lifestyle & Prolonged steroid & \\
$\begin{array}{l}\text { Post-transplant weight gain } \\
\text { Diabetes, Hypertension }\end{array}$ & Increased fat and fructose intake & \\
$\begin{array}{l}\text { Dyslipedemia } \\
\text { Increasing age }\end{array}$ & Alcohol intake & \\
Sleep apnea & & Calcineurin inhibitors \\
Alcohol & & Sirolimus \\
\hline
\end{tabular}

Prevention of NAFLD/NASH post Liver Transplantation

Modification of recipient factors

Modification of recipient factois Pretransplant:

- Optimization of treatment of DM Hypertension, Dyslipedemia

- Weight control measures: Diet(high protein, low fat and low fructose)and regular exercises

Post liver Transplantation:

- Avoid excessive weight gain: More protein, less fat and carbohydrate

- Regular exercises

- Management of DM, Hypertension and dyslipedemia with medications as before LT

- Use of insulin sensitizers like metformin and antioxidant (Vitamin E)where ever indicated
Immunosuppression modifications

- Avoidance of steroid or early discontinuation within 3 months

- Minimization of $\mathrm{CNI}$ to prevent components of metabolic syndrome

Figure 1 Prevention of NAFLD post liver transplant. NAFLD, non-alcoholic fatty liver disease.

for de novo or recurrent NAFLD (21).

Interactions between donor and recipient genetics may also affect disease recurrence. Gene variants, such as the patatin-like phospholipase domain-containing 3 (PNPLA3) and transmembrane 6 superfamily member 2 (TM6SF2) E167K, which are contributory to hepatic triglyceride remodeling and interference of very-lowdensity lipoprotein secretion have been studies in few studies (22). These genetic alleles confer susceptibility for the risk of fibrosis and steatosis. There is no known association between donor clinical risk factors for hepatic steatosis and the risk for recurrent or de novo hepatic steatosis in the transplanted liver graft $(22,23)$.

\section{Management of post liver transplant NAFLD}

There are few studies available on the management of $d e$ novo or recurrent NAFLD after liver transplant. Majority of recommendation are based on studies done on patient who do not have LT. As in pre-transplant patients no definitive pharmacologic therapies are available for the treatment of hepatic steatosis developed post-transplant (recurrent or de novo) (Figure 1).

Prevention is better than cure so the management of metabolic components of MS after transplant should start with prevention.

\section{Weight reduction}

Intervention to prevent weight gain may be more successful than weight loss attempts after LT. Avoiding weight gain have long-term benefits in reduction of hypertension, hyperlipidemia and diabetes with subsequent reduction in cardiovascular disease (CVD), renal dysfunction 
and fatty liver $(24,25)$. However, the right timing for implementation of lifestyle modification in recurrent or de novo NAFLD post LT has yet to be determined. It has been shown in non-transplant cohorts of patients with NAFLD, that lifestyle changes associated with weight loss of $10 \%$ can reduce steatohepatitis and regress hepatic fibrosis in $47 \%$, and $19 \%$, respectively (25). In terms of physical activity, both aerobic and resistance training are beneficial in reducing steatosis and necroinflammation in NAFLD patients. However large studies in post-transplant setting are not available. In a study by Krasnoff et al. (26) liver transplanted patients $(\mathrm{n}=151)$ were randomized to exercise and dietary counselling or usual care. Patients randomized to exercise and dietary counselling showed a similar increase in body weight, fat mass and lean mass compared with the control group. However, only one third of patients showed complete adherence to exercise group. Fully adherent patients did show improvements in VO2max (maximum oxygen consumption) and body composition when compared to nonadherent patients. The maximum weight gain occurs in the first year post LT; hence early intervention to prevent weight gain in the first year of LT is best time to prevent liver steatosis.

\section{Key points}

* Early prevention of weight gain should be advised in all transplant recipients; in particular those with NASH related LT;

* Lifestyle modifications like avoiding alcohol, smoking, excessive fructose intake in forms of cola drinks, low carbohydrate diet and regular exercises should represent the first-line therapy in patients with MS and/or recurrent or de novo NAFLD/NASH after LT;

* In patients who are overweight or obese after LT, a weight loss of $7 \%$ to $10 \%$ should be pursued with low calorie diet and aerobic and resistance physical activity as it helps in patients who have NASH without liver transplant;

* A multidisciplinary approach which includes primary physician, dietician, transplant coordinator and patient councillor is advisable after LT to prevent and correct post LT steatosis.

Bariatric surgery had shown promising results in patients with NASH to reverse steatosis, inflammation and fibrosis. In a meta-analysis done by Mummadi et al. (27) in patients with NAFLD (non-LT population) who underwent bariatric surgery showed reduction in BMI $(>10 \%)$ was correlated with improvement of steatosis in $92 \%$, steatohepatitis in $81 \%$ and fibrosis in $66 \%$ of patients. Complete resolution of NASH was seen in $70 \%$ of patient's post-bariatric surgery. It was concluded that bariatric surgery is helpful in reversibility of NAFLD.

Patients who were obese and underwent a simultaneous bariatric surgery and LT shows less weight gain and hepatic steatosis post LT. In a study 37 patients with BMI $>35$ who underwent only LT and weight loss prior to LT were compared with 7 patients who underwent LT with sleeve gastrectomy. There was a higher weight gain, steatosis, post-transplant diabetes, graft loss and death in patients who underwent only LT when compared with patients who underwent LT and simultaneous sleeve gastrectomy. This proof of concept study indicates promising role of simultaneous bariatric surgery in obese patients undergoing transplant to prevent hepatic steatosis and weight gain post LT. Hence weight reduction or prevention of weight gain remains the cornerstone of preventive and corrective method to control post LT recurrence of NAFLD (28). Routine use of simultaneous sleeve gastrectomy in order to prevent hepatic steatosis in obese liver transplant patients has yet to be proven in large studies.

\section{Pharmacotherapy}

There is paucity of studies for management of NAFLD in patients after LT. Hence pharmacological management of post-transplant NAFLD/NASH is not different from management of NAFLD in non-transplant patients. Though treatment could be extrapolated as in non-transplanted patients, various factors need to address in patients after LT like dose and duration of immunosuppression used, interaction with various drugs, transplant complications, donor related factors and genetics of recipient. Renal complications, graft rejection and metabolic complications related to immunosuppression will affect the drugs used for the treatment of NAFLD. Hence drugs used in nontransplant patients may not be effective in patients post LT. Hypertension management is same as in general population and general principles like sodium restriction, smoking cessation, alcohol avoidance and exercise, are first line interventions. Obstructive sleep apnea associates strongly with obesity and hypertension so should be assessed and treated as well.

The use of insulin sensitizers medications remains the cornerstone of management of NAFLD like metformin and peroxisome proliferator-activated receptor agonist like 
pioglitazone (4). Metformin improves aminotransferases in few studies; however pooled results have found no significant improvement in NASH. This metaanalysis concluded little evidence to support the use of metformin in patients with NAFLD without diabetes (29). Thiazolidinediones (TZDs), including Pioglitazone was evaluated in a large landmark study by Sanyal et al. (30) in which 247 adults with nonalcoholic steatohepatitis and without diabetes to receive pioglitazone at a dose of 30 $\mathrm{mg}$ daily (80 subjects), vitamin $\mathrm{E}$ at a dose of 800 IU daily (84 subjects), or placebo (83 subjects), for 96 weeks. Both Vitamin E and Pioglitazone demonstrated improvement in different components of NASH like hepatic steatosis, ballooning and inflammation however only Vitamin E showed statistically significance. Liver fibrosis did not achieve any significant reduction in either group. Prolonged use of Vitamin E may increase risk of malignancy like prostate cancer in some studies and found to have no protective effect in others, so its prolonged use especially in elderly patients needs further data (31,32). Vitamin E and pioglitazone may have a role in NAFLD in non-diabetic pretransplant patient but no data exist on its use in liver transplant recipients. Transplant recipients are at higher risk of CVD and malignancies and both of these agents have adverse risk profiles including weight gain, CVD and malignancies (33) so there use in post-transplant NAFLD patient needs further data. Liraglutide, a glucagon-like peptide-1 analog, shows promise in NAFLD/NASH pretransplant, however its safety even in pre transplant NASH has not been fully evaluated. Its use in posttransplant NAFLD has not been evaluated (34).

Statins has been investigated for the treatment of NAFLD with mixed results, so American association for the study of liver has not recommended use of statins in the treatment of NAFLD except for the treatment of dyslipidemia. Similarly, statins have not been evaluated in the treatment of NASH post LT (4).

\section{Immunosuppression protocols}

Immunosuppression regimens after LT influence the metabolic profile of liver transplant recipients. Patients should be carefully monitored for the recurrence of MS and NAFLD especially in patients with NASH related cirrhosis.

Corticosteroids and CNI are associated with increased risk of development of weight gain, diabetes, hypertension and dyslipidemia. Hence dose and duration of steroids should be minimised during maintenance phase of immunosuppression. In a meta-analysis no significant difference was seen in graft loss, infection rates and overall survival in patients with steroid-free regimens versus steroid-based immunosuppression (35). Hence steroid should be either avoided or to be used to minimum duration. Few studies have shown allograft steatosis with use of tacrolimus. Sirolimus in combination with CNI has been associated with dyslipidemia, insulin signaling pathway alteration and steatosis $(36,37)$. Use of prebiotics and probiotics have limited use in the management of NAFLD pre and post-transplant.

\section{Recommendations for prevention of NAFLD/NASH post LT}

* Early prevention of weight gain should be advised in all transplant recipients; in particular those with NASH related LT;

* Lifestyle modifications like avoiding alcohol, smoking, excessive fructose intake in forms of cola drinks, low carbohydrate diet and regular exercises should represent the first-line therapy in patients with MS and/or recurrent or de novo NAFLD/NASH after LT;

* Till we have more data on use of drugs in patients with post LT and NAFLD/NASH, no single drug can be recommended for prevention/ treatment for recurrence of post-transplant NAFLD/NASH;

* Drugs used to treat components of MS like hypertension, dyslipidemia and diabetes can be used in the transplant recipient with NAFLD/NASH to prevent future complications related to these diseases;

- Patients transplanted for NASH-related cirrhosis, immunosuppression with steroids should be minimised for in dosages and duration;

* CNI minimization or alteration may be tried in patients who develop components of MS like dyslipidemia, hypertension or insulin resistance.

\section{Summary}

NASH related cirrhosis and LT is growing all over the world with rising incidence of diabetes and obesity. LT is the definitive cure for complications related to NASH related cirrhosis. However metabolic factors which were commonly present in patients with NASH related cirrhosis worsen after LT due to immunosuppressive regimen. As there are no trials in managing NAFLD/NASH post LT most of data are extrapolated from trials used in patient 
with NASH who do not have LT.

NAFLD and components of MS prior to LT like obesity, hypertension, hyperlipidemia, diabetes continue to play an important role in development of hepatic steatosis posttransplant. Life style modifications, prevention of weight gain, no or minimization of steroid use, simultaneous bariatric surgery in obese patients still remains the cornerstone of managing patients with NASH both pre and post LT. Use of metformin, statins, insulin sensitizers, vitamin $\mathrm{E}$ all have been tried with limited success in patients with NASH before transplantation and are being used after LT in patients with NASH. Many emerging therapies for NAFLD are currently under investigation and require further investigation in large randomized trials.

\section{Acknowledgments}

Funding: None.

\section{Footnote}

Conflicts of Interest: The authors have no conflicts of interest to declare.

Ethical Statement: The authors are accountable for all aspects of the work in ensuring that questions related to the accuracy or integrity of any part of the work are appropriately investigated and resolved.

Open Access Statement: This is an Open Access article distributed in accordance with the Creative Commons Attribution-NonCommercial-NoDerivs 4.0 International License (CC BY-NC-ND 4.0), which permits the noncommercial replication and distribution of the article with the strict proviso that no changes or edits are made and the original work is properly cited (including links to both the formal publication through the relevant DOI and the license). See: https://creativecommons.org/licenses/by-ncnd/4.0\%.

\section{References}

1. Torres DM, Williams CD, Harrison SA. Features, diagnosis, and treatment of nonalcoholic fatty liver disease. Clin Gastroenterol Hepatol 2012;10:837-58.

2. Vernon G, Baranova A, Younossi ZM. Systematic review: the epidemiology and natural history of non-alcoholic fatty liver disease and non-alcoholic steatohepatitis in adults.
Aliment Pharmacol Ther 2011;34:274-85.

3. Ong JP, Elariny H, Collantes R, et al. Predictors of nonalcoholic steatohepatitis and advanced fibrosis in morbidly obese patients. Obes Surg 2005;15:310-5.

4. Chalasani N, Younossi Z, Lavine JE, et al. The diagnosis and management of non-alcoholic fatty liver disease: practice Guideline by the American Association for the Study of Liver Diseases, American College of Gastroenterology, and the American Gastroenterological Association. Hepatology 2012;55:2005-23.

5. Charlton MR, Burns JM, Pedersen RA, et al. Frequency and outcomes of liver transplantation for nonalcoholic steatohepatitis in the United States. Gastroenterology 2011;141:1249-53.

6. Sprinzl MF, Weinmann A, Lohse N, et al. Metabolic syndrome and its association with fatty liver disease after orthotopic liver transplantation. Transpl Int 2013;26:67-74.

7. Dumortier J, Giostra E, Belbouab S, et al. Non-alcoholic fatty liver disease in liver transplant recipients: another story of "seed and soil." Am J Gastroenterol 2010;105:613-20.

8. Dureja P, Mellinger J, Agni R, et al. NAFLD recurrence in liver transplant recipients. Transplantation 2011;91:684-9.

9. Patil DT, Yerian LM. Evolution of nonalcoholic fatty liver disease recurrence after liver transplantation. Liver Transpl 2012;18:1147-53.

10. Seo S, Maganti K, Khehra M, et al. De novo nonalcoholic fatty liver disease after liver transplantation. Liver Transpl 2007;13:844-7.

11. Vallin M, Guillaud O, Boillot O, et al. Recurrent or de novo non-alcoholic fatty liver disease after liver transplantation: natural history based on liver biopsy analysis. Liver Transpl 2014;20:1064-71.

12. Malik SM, Devera ME, Fontes P, et al. Recurrent disease following liver transplantation for nonalcoholic steatohepatitis cirrhosis. Liver Transpl 2009;15:1843-51.

13. Galvin Z, Rajakumar R, Chen E, et al. Predictors of De Novo Nonalcoholic Fatty Liver Disease After Liver Transplantation and Associated Fibrosis. Liver Transpl 2019;25:56-67.

14. Yalamanchili K, Saadeh S, Klintmalm GB, et al. Nonalcoholic fatty liver disease after liver transplantation for cryptogenic cirrhosis or non-alcoholic fatty liver disease. Liver Transpl 2010;16:431-9.

15. Pagadala M, Dasarathy S, Eghtesad B, et al. Posttransplant metabolic syndrome: an epidemic waiting to happen. Liver Transpl 2009;15:1662-70.

16. Gisbert C, Prieto M, Berenguer M, et al. Hyperlipidemia 
in liver transplant recipients: prevalence and risk factors. Liver Transpl Surg 1997;3:416-22.

17. Watt KD, Charlton MR. Metabolic syndrome and liver transplantation: a review and guide to management. J Hepatol 2010;53:199-206.

18. Li DW, Lu TF, Hua XW, et al. Risk factors for new onset diabetes mellitus after liver transplantation: a metaanalysis. World J Gastroenterol 2015;21:6329-40.

19. Iadevaia M, Giusto M, Giannelli V, et al. Metabolic syndrome and cardiovascular risk after liver transplantation: a single-center experience. Transplant Proc 2012;44:2005-6.

20. Zimmermann A, Zobeley C, Weber MM, et al. Changes in lipid and carbohydrate metabolism under mTOR- and calcineurin-based immunosuppressive regimen in adult patients after liver transplantation. Eur J Intern Med 2016;29:104-9.

21. Firpi RJ, Tran TT, Flores P, et al. Sirolimus-induced hyperlipidaemia in liver transplant recipients is not dosedependent. Aliment Pharmacol Ther 2004;19:1033-9.

22. Romeo S, Kozlitina J, Xing C, et al. Genetic variation in PNPLA3 confers susceptibility to nonalcoholic fatty liver disease. Nat Genet 2008;40:1461-5.

23. Dongiovanni P, Valenti L, Rametta R, et al. Genetic variants regulating insulin receptor signalling are associated with the severity of liver damage in patients with non-alcoholic fatty liver disease. Gut 2010;59:267-73.

24. Wong VW, Chan RS, Wong GL, et al. Community-based lifestyle modification programme for non-alcoholic fatty liver disease: a randomized controlled trial. J Hepatol 2013;59:536-42.

25. Vilar-Gomez E, Martinez-Perez Y, Calzadilla-Bertot L, et al. Weight loss through lifestyle modification significantly reduces features of nonalcoholic steatohepatitis. Gastroenterology 2015;149:367-78.

26. Krasnoff JB, Vintro AQ, Ascher NL, et al. A randomized trial of exercise and dietary counseling after liver transplantation. Am J Transplant. 2006;6:1896-905.

27. Mummadi RR, Kasturi KS, Chennareddygari S, et al.

doi: $10.21037 / \operatorname{tgh} .2020 .03 .02$

Cite this article as: Sharma P, Arora A. Approach to prevention of non-alcoholic fatty liver disease after liver transplantation. Transl Gastroenterol Hepatol 2020;5:51.
Effect of bariatric surgery on nonalcoholic fatty liver disease: systematic review and meta-analysis. Clin Gastroenterol Hepatol 2008;6:1396-402.

28. Heimbach JK, Watt KD, Poterucha JJ, et al. Combined liver transplantation and gastric sleeve resection for patients with medically complicated obesity and end-stage liver disease. Am J Transplant 2013;13:363-8.

29. Musso G, Gambino R, Cassader M, et al. A metaanalysis of randomized trials for the treatment of non-alcoholic fatty liver disease. Hepatology 2010;52:79-104.

30. Sanyal AJ, Chalasani N, Kowdley KV, et al. Pioglitazone, vitamin $\mathrm{E}$, or placebo for nonalcoholic steatohepatitis. $\mathrm{N}$ Engl J Med 2010;362:1675-85.

31. Klein EA, Thompson IM Jr, Tangen CM et al. Vitamin $\mathrm{E}$ and the risk of prostate cancer: the Selenium and Vitamin E Cancer Prevention Trial (SELECT). JAMA 2011;306:1549-56.

32. Wang L, Sesso HD, Glynn RJ et al. Vitamin E and C supplementation and risk of cancer in men: posttrial follow-up in the Physicians' Health Study II randomized trial. Am J Clin Nutr 2014;100:915-23.

33. Miller ER, Pastor-Barriuso R, Dalal D, et al. Meta-analysis: high-dosage vitamin $\mathrm{E}$ supplementation may increase allcause mortality. Ann Intern Med 2005;142:37-46.

34. Armstrong MJ, Gaunt P, Aithal GP, et al. Liraglutide safety and efficacy in patients with non-alcoholic steatohepatitis (LEAN): a multicentre, double-blind, randomised, placebo-controlled phase 2 study. Lancet 2016;387:679-90.

35. Segev DL, Sozio SM, Shin EJ, et al. Steroid avoidance in liver transplantation: meta-analysis and meta-regression of randomized trials. Liver Transpl 2008;14:512-25.

36. Neff GW, Montalbano M, Tzakis AG. Ten years of sirolimus therapy in orthotopic liver transplant recipients. Transplant Proc 2003;35:209S-16S.

37 Contos MJ, Cales W, Sterling RK, et al. Development of non-alcoholic fatty liver disease after orthotopic liver transplantation for cryptogenic cirrhosis. Liver Transpl 2001;7:363-73. 